\title{
Spectral properties and the accuracy of mean-field approaches for epidemics on correlated power-law networks
}

\author{
Diogo H. Silva $\odot,{ }^{1}$ Silvio C. Ferreira $\odot,{ }^{1,2}$ Wesley Cota $\odot,{ }^{1}$ Romualdo Pastor-Satorras,${ }^{3}$ and Claudio Castellano $\odot^{4}$ \\ ${ }^{1}$ Departamento de Física, Universidade Federal de Viçosa, 36570-900 Viçosa, Minas Gerais, Brazil \\ ${ }^{2}$ National Institute of Science and Technology for Complex Systems, 22290-180 Rio de Janeiro, Rio de Janeiro, Brazil \\ ${ }^{3}$ Departament de Física, Universitat Politècnica de Catalunya, Campus Nord B4, 08034 Barcelona, Spain \\ ${ }^{4}$ Istituto dei Sistemi Complessi (ISC-CNR), Consiglio Nazionale delle Ricerche, Via dei Taurini 19, 00185 Roma, Italy
}

(Received 5 July 2019; published 15 October 2019)

\begin{abstract}
We present a comparison between stochastic simulations and mean-field theories for the epidemic threshold of the susceptible-infected-susceptible model on correlated networks (both assortative and disassortative) with a power-law degree distribution $P(k) \sim k^{-\gamma}$. We confirm the vanishing of the threshold regardless of the correlation pattern and the degree exponent $\gamma$. Thresholds determined numerically are compared with quenched mean-field (QMF) and pair quenched mean-field (PQMF) theories. Correlations do not change the overall picture: The QMF and PQMF theories provide estimates that are asymptotically correct for large sizes for $\gamma<5 / 2$, while they only capture the vanishing of the threshold for $\gamma>5 / 2$, failing to reproduce quantitatively how this occurs. For a given size, PQMF theory is more accurate. We relate the variations in the accuracy of QMF and PQMF predictions with changes in the spectral properties (spectral gap and localization) of standard and modified adjacency matrices, which rule the epidemic prevalence near the transition point, depending on the theoretical framework. We also show that, for $\gamma<5 / 2$, while QMF theory provides an estimate of the epidemic threshold that is asymptotically exact, it fails to reproduce the singularity of the prevalence around the transition.
\end{abstract}

DOI: 10.1103/PhysRevResearch.1.033024

\section{INTRODUCTION}

Metabolic chains of protein interactions [1], collaborations among scientists, co-starring in a movie [2], and person-toperson contacts [3] are all examples of interacting systems that can be modeled using complex networks [2]. A large number of networks representing real systems show a heavy-tailed degree distribution described by a power law $P(k) \sim k^{-\gamma}$ $[4,5]$, usually with strong levels of correlations [6,7]. Degree correlations are encoded in the conditional probability $P\left(k^{\prime} \mid k\right)$ that a vertex of degree $k$ is connected to a vertex of degree $k^{\prime}$ [6]. Technological networks, such as the Internet, show in general disassortative mixing [6,7], i.e., vertices of large degree tend to be connected with those of small degree and vice versa. Assortative mixing occurs in social networks, where connections preferentially occur among vertices exhibiting similar degree. Since uncorrelated networks usually simplify theoretical approaches, they are typical benchmarks for the investigation of dynamical processes on networks and have been considered in many studies [8-10]. However, the ubiquitousness of correlations in real networks naturally calls for the investigation of the effect of correlated interaction patterns. While the effects of degree correlations have been considered for several dynamical processes [11-16], a full understanding

Published by the American Physical Society under the terms of the Creative Commons Attribution 4.0 International license. Further distribution of this work must maintain attribution to the author(s) and the published article's title, journal citation, and DOI. of their effects on the performance of theoretical approaches is still missing.

A basic approach to investigate dynamical processes on networks is the heterogeneous mean-field (HMF) theory, in which degree heterogeneity and correlations are taken into account through the distributions $P(k)$ and $P\left(k \mid k^{\prime}\right)$, respectively $[8,9,17,18]$. A more refined approach is provided by the quenched mean-field (QMF) theory [19-21], which considers the full topology as described by the unweighted adjacency matrix (defined as $A_{i j}=1$ if vertices $i$ and $j$ are connected and $A_{i j}=0$ otherwise) and thus takes into account the detailed connectivity structure.

A crucial question in this context is the ability of theories to accurately predict the epidemic threshold of the susceptibleinfected-susceptible (SIS) dynamics, the most basic epidemic process with an absorbing-state phase transition [21-28]. For random uncorrelated networks, such as those created according to the uncorrelated configuration model [29], when $\gamma<$ $5 / 2$ the two theories tend to agree, predicting a vanishing threshold as the network size diverges [22]. For $\gamma>3$ instead, QMF theory correctly predicts again the asymptotic vanishing of the epidemic threshold [30], while the HMF theory fails, predicting the existence of a finite threshold. In spite of being qualitatively correct, QMF theory is however not able to accurately predict the effective finite-size epidemic threshold in this case [25]. A further quantitative improvement of the QMF theory has been achieved in Ref. [26] [hereafter called of pair QMF (PQMF) theory] by means of the explicit inclusion of pairwise dynamical correlations [31-33]. See [34] for a recent application of the PQMF theory in epidemic containment. 
In this work we investigate the ability of the aforementioned approaches (HMF, QMF, and PQMF) to quantitatively predict the value of the epidemic threshold for both uncorrelated and correlated networks generated using the Weber-Porto model [35] and for real-world topologies. We find that correlations do not change qualitatively the scenario found on uncorrelated networks. The epidemic threshold vanishes asymptotically with the system size for both assortative and disassortative correlations. For $\gamma<5 / 2$, both QMF and PQMF theories seem to provide an asymptotically exact estimate of the numerical threshold, while they are only qualitatively correct for $\gamma>5 / 2$. As in the case of uncorrelated networks [26], PQMF theory outperforms the other theories. The amplitude of the discrepancies between numerics and theory is correlated with violations of the assumptions underlying them, revealing that both theories tend to be more accurate if the principal eigenvector of the (effective) adjacency matrix is not strongly localized or the spectral gap is large. The same scenario is found to hold when SIS dynamics is considered on a set of real-world topologies. In addition, we analyze the singularity of the prevalence near the transition point through the critical exponent $\beta$, defined as $\rho \sim\left(\lambda-\lambda_{c}\right)^{\beta}$. Interestingly, we find that for $\gamma<5 / 2$, even if the QMF theory provides an asymptotically exact estimate of the position of the epidemic threshold, the QMF prediction for the prevalence exponent, $\beta^{(\mathrm{QMF})}=1[23,36]$, is correct only not too close to the transition.

The rest of the paper is organized as follows. Section II describes the models used to generate correlated heavy-tailed networks, the implementation of the SIS model, and the theoretical approaches. A comparison between simulations and theory on synthetic and real networks in presented in Sec. III. A summary is given and conclusions are drawn in Sec. IV. The Appendix summarizes the properties of the real networks investigated.

\section{MODELS AND METHODS}

\section{A. Weber-Porto configuration model}

The degree correlations encoded in the conditional probability $P\left(k^{\prime} \mid k\right)$ can be more easily interpreted by the simple metrics of the average degree of the nearest neighbors as a function of the vertex degree [6], defined as

$$
\kappa_{\mathrm{nn}}(k)=\sum_{k^{\prime}=k_{\min }}^{k_{\max }} k^{\prime} P\left(k^{\prime} \mid k\right),
$$

where $k_{\min }$ and $k_{\max }$ are the lower and upper cutoffs of the degree distribution. If $\kappa_{\mathrm{nn}}(k)$ increases or decreases with $k$, the networks are assortative or disassortative, respectively. In the case of uncorrelated networks we have [6]

$$
P\left(k^{\prime} \mid k\right)=P_{\mathrm{e}}\left(k^{\prime}\right)=k^{\prime} P\left(k^{\prime}\right) /\langle k\rangle,
$$

which implies that $\kappa_{\mathrm{nn}}=\left\langle k^{2}\right\rangle /\langle k\rangle=\langle k\rangle_{\mathrm{e}}$ does not depend on $k$. We use here the edge distribution average $\langle A(k)\rangle_{\mathrm{e}}=$ $\sum_{k} A(k) P_{\mathrm{e}}(k)$, where $P_{\mathrm{e}}(k)$ is the probability that an edge ends on a vertex of degree $k$.

We are interested in heavy-tailed networks with degree distribution $P(k) \sim k^{-\gamma}$ and correlation given by $\kappa_{\mathrm{nn}}(k) \sim k^{\alpha}$. These networks can be generated using an algorithm proposed

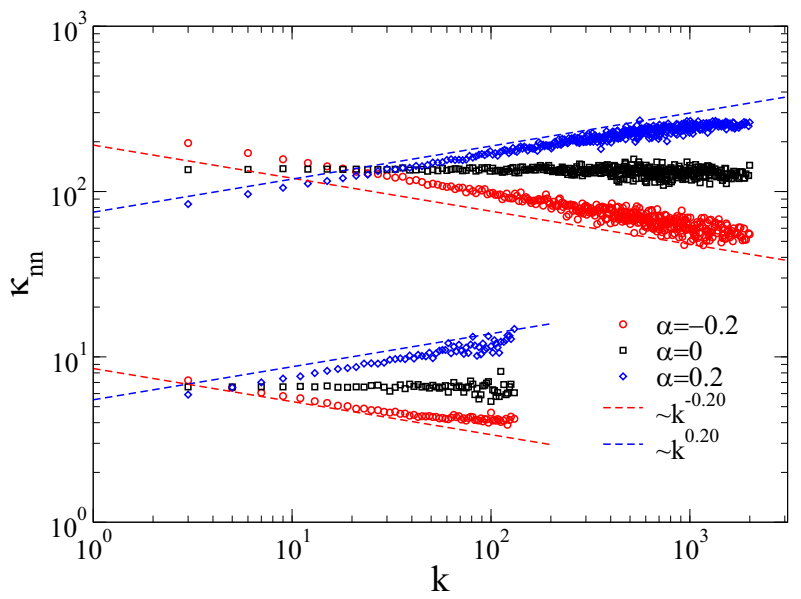

FIG. 1. Average degree of the nearest neighbors as a function of the degree for networks built with the WPCM algorithm [35] for power-law degree distributions with $\gamma=2.3$ (top curves) and $\gamma=3.5$ (bottom curves). The network size is $N=10^{6}$ and the lower cutoff is $k_{\min }=3$. The upper cutoff is given by $k_{\max }=2 \sqrt{N}$ for $\gamma=2.3$ and $N P\left(k_{\max }\right)=1$ for $\gamma=3.5$.

by Weber and Porto [35], hereafter called the Weber-Porto configuration model (WPCM). The degree of each vertex is drawn according to the degree distribution $P(k)$ and initially each node has $k$ unconnected stubs. Two stubs are randomly chosen and connected with probability

$$
P_{\text {link }}\left(q^{\prime}, q\right)=\frac{f\left(q^{\prime}, q\right)}{f_{\max }},
$$

where $q$ and $q^{\prime}$ are the respective degrees of the chosen vertices and $f_{\max }$ is the maximum value of

$$
f\left(q, q^{\prime}\right)=1+\frac{\left[\kappa_{\mathrm{nn}}(q)-\langle k\rangle_{\mathrm{e}}\right]\left[\kappa_{\mathrm{nn}}\left(q^{\prime}\right)-\langle k\rangle_{\mathrm{e}}\right]}{\left\langle k \kappa_{\mathrm{nn}}\right\rangle_{\mathrm{e}}-\langle k\rangle_{\mathrm{e}}^{2}},
$$

computed over the whole network. Self- and multiple connections are forbidden. In the absence of degree correlations, we have $\kappa_{\mathrm{nn}}=\langle k\rangle_{\mathrm{e}}$, implying $f\left(q, q^{\prime}\right)=1$ and $P_{\text {link }}=1$. See Ref. [35] for more details.

Figure 1 shows $\kappa_{\mathrm{nn}}$ as a function of $k$ for networks obtained with the WPCM algorithm [35] using different values of $\gamma$ and $\alpha$, with lowest degree $k_{\min }=3$. We adopt different upper cutoffs for the degree distribution. For $\gamma<3$, the structural cutoff $k_{\max }=2 \sqrt{N}$ [37] is used, while for $\gamma>3$, a rigid cutoff is determined by the condition $N P\left(k_{\max }\right)=1$ [38]. The first choice allows us to enhance the effects of hubs and to approach faster the thermodynamic limit while fulfilling the criterion $k_{\max }<\sqrt{\langle k\rangle N}$ necessary to produce uncorrelated networks in the case $\alpha=0$ [37]. The second choice is justified by numerical reasons explained in Sec. II B. The predetermined scaling law $\kappa_{\mathrm{nn}}(k) \sim k^{\alpha}$ is very well reproduced. Small deviations for positive or negative $\alpha$ are due to the network finite size that prevents $\kappa_{\mathrm{nn}}$ from decaying or increasing indefinitely with $k$. The range of the power-law behavior is extended as the network size increases. 


\section{B. SIS simulations}

In the SIS model, each edge of an infected vertex transmits the epidemic with rate $\lambda$, while infected nodes recover spontaneously with constant rate $\mu$. The latter is fixed to $\mu=1$ without loss of generality. The model can be simulated with the optimized Gillespie scheme proposed in Ref. [22]. See also Ref. [39] for more details.

We consider quasistationary simulations [40] in which the dynamics returns to a previously visited active configuration whenever the absorbing state, consisting of all vertices susceptible, is visited. This strategy permits us to circumvent the difficulties of dealing with the absorbing state, which is the only true stationary state for any finite-size networks. More details can be found in Refs. [39,41].

The effective transition point $\lambda_{c}(N)$, above which the epidemic remains in an active phase for very long periods, can be estimated using the position of the maximum of the dynamical susceptibility [22]

$$
\psi=N \frac{\left\langle\rho^{2}\right\rangle-\langle\rho\rangle^{2}}{\langle\rho\rangle} .
$$

The choice of structural (for $\gamma<3$ ) and rigid (for $\gamma>3$ ) upper cutoffs allows the determination of the epidemic threshold unambiguously, avoiding multiple peaks and the smearing of the transition that can appear for SIS on power-law degree distribution networks, especially with large values of $\gamma[22,27]$.

\section{Mean-field theories for correlated networks}

In this section we summarize the predictions of the theoretical approaches that will be compared to numerical simulations in Sec. III. For QMF and PQMF approaches, the equations for uncorrelated and correlated networks are formally the same: Correlations have only the effect of modifying the entries of the adjacency matrix $A_{i j}$.

\section{Correlated heterogeneous mean-field theory}

Heterogeneous mean-field theory takes into account nearest-neighbor correlations by the explicit consideration of the conditional probability $P\left(k^{\prime} \mid k\right)$. The HMF equation for the density of infected vertices with degree $k, \rho_{k}$, is given by [42]

$$
\frac{d \rho_{k}}{d t}=-\rho_{k}+\left(1-\rho_{k}\right) \lambda \sum_{l} k P(l \mid k) \rho_{l},
$$

which yields an epidemic threshold given by

$$
\lambda_{c} \Upsilon^{(1)}=1,
$$

where $\Upsilon^{(1)}$ is the largest eigenvalue of the connectivity matrix $C_{k l}=k P(l \mid k)$. For WPCM networks we have $P(l \mid k)=$ $P_{\mathrm{e}}(l) f(l, k)$, and therefore

$$
C_{k l}=\frac{k l P(l)}{\langle k\rangle} f(l, k) .
$$

In the absence of correlations, $C_{k l}=\frac{k l P(l)}{\langle k\rangle}$, implying that $\lambda_{c}=\frac{\langle k\rangle}{\left\langle k^{2}\right\rangle}[11,42]$. It has been shown [11] that the HMF threshold vanishes for scale-free networks with $2<\gamma<3$ in the thermodynamic limit, irrespective of degree correlations.

\section{Quenched mean-field theory}

According to the QMF theory, which neglects pairwise dynamical correlations, the evolution of the probability $\rho_{i}$ that a vertex $i$ is infected is given by [20]

$$
\frac{d \rho_{i}}{d t}=-\rho_{i}+\lambda\left(1-\rho_{i}\right) \sum_{j=1}^{N} A_{i j} \rho_{j},
$$

where $N$ is the network size. The epidemic threshold is given by

$$
\lambda_{\mathrm{c}}^{\mathrm{QMF}} \Lambda^{(1)}=1,
$$

where $\Lambda^{(1)}$ is the largest eigenvalue (LEV) of the adjacency matrix $A_{i j}$. In the steady state we have

$$
\rho_{i}=\frac{\lambda \sum_{j} A_{i j} \rho_{j}}{1+\lambda \sum_{j} A_{i j} \rho_{j}} .
$$

Using Eq. (11), Goltsev et al. [23] have shown that $\rho_{i} \sim v_{i}^{(1)}$ for $\lambda \gtrsim \lambda_{c}^{\mathrm{QMF}}$, where $\left\{v_{i}^{(1)}\right\}$ is the principal eigenvector (PEV) corresponding to the LEV of $A_{i j}, \sum_{i} A_{i j} v_{j}^{(1)}=\Lambda^{(1)} v_{i}^{(1)}$. Thus, the order parameter $\rho=\sum_{i} \rho_{i} / N$ of the QMF theory vanishes at $\lambda_{c}^{\mathrm{QMF}}$ as

$$
\rho \simeq a_{1}\left(\lambda \Lambda^{(1)}-1\right)
$$

where

$$
a_{1}(N)=\frac{\sum_{i=1}^{N} v_{i}^{(1)}}{N \sum_{i=1}^{N}\left[v_{i}^{(1)}\right]^{3}} .
$$

This same result was obtained independently in Ref. [36].

Within the QMF framework, Eq. (12) works well, close to the threshold $\lambda_{c}^{\mathrm{QMF}}$, under the hypothesis that the network presents a spectral gap, i.e., the second largest eigenvalue of $A_{i j}$ is much smaller than the first, $\Lambda^{(1)} \gg \Lambda^{(2)}$. According to Eqs. (12) and (13), the QMF theory predicts the existence of an endemic state, with a finite fraction of infected vertices above the threshold $\lambda_{c}^{\mathrm{QMF}}=1 / \Lambda^{(1)}$, only if $a_{1} \sim O(1)$, which occurs when the PEV is delocalized. Localization can be quantified by the inverse participation ratio (IPR) for the normalized PEV [23], defined as

$$
Y_{4}=\sum_{i=1}^{N}\left[v_{i}^{(1)}\right]^{4}
$$

If the PEV is delocalized then $Y_{4} \sim N^{-1}$, while $Y_{4} \sim O(1)$ if the $\mathrm{PEV}$ is localized on a finite number of vertices, but weaker forms of localization can be observed [43].

For random uncorrelated power-law networks the PEV is always localized [43]. For $\gamma<5 / 2$ it is (weakly) localized on a subextensive set of nodes coinciding with the maximum $K$-core, a subgraph of strongly mutually interconnected nodes with degree larger than or equal to $K$ [44]. In such a case $Y_{4} \sim N^{(\gamma-3) / 2}$. For $\gamma>5 / 2$ it is instead strongly localized on the largest hub plus its nearest neighbors and $Y_{4} \sim O(1)$ [45]. Hence, within QMF theory the threshold separates the absorbing phase from an active but strictly nonendemic state. However this does not imply that QMF predictions are necessarily flawed. Equation (9) factorizes the state of nearest neighbors and thus neglects dynamical correlations 
among them. These dynamical correlations actually transmit the infection from the localized PEV to the rest of the network and thus may in principle transform the active but localized state just above $\lambda_{c}^{\mathrm{QMF}}$ into a full-fledged endemic state $[25,46]$.

\section{Pair quenched mean-field theory}

An improvement with respect to QMF theory is obtained by taking into account some dynamical correlations using the pairwise approximation developed in Ref. [26], where all derivation details can be found. Consider the probability $\phi_{i j}$ that a vertex $i$ is susceptible and a neighbor $j$ is infected. The dynamical system to be solved is

$$
\frac{d \rho_{i}}{d t}=-\rho_{i}+\lambda \sum_{j} \phi_{i j} A_{i j}
$$

and

$$
\begin{aligned}
\frac{d \phi_{i j}}{d t}= & -(2+\lambda) \phi_{i j}+\rho_{j}+\lambda \sum_{l} \frac{\omega_{i j} \phi_{j l}}{1-\rho_{j}}\left(A_{j l}-\delta_{i l}\right) \\
& -\lambda \sum_{l} \frac{\phi_{i j} \phi_{i l}}{1-\rho_{i}}\left(A_{i l}-\delta_{l j}\right),
\end{aligned}
$$

where $\omega_{i j}=1-\phi_{i j}-\rho_{i}$.

Here we develop a bit further the theory to analyze the steady state near the critical point. Keeping only leading terms up to second order in $\rho_{i}$ in Eq. (16), we obtain

$$
\phi_{i j} \approx \frac{(2+\lambda) \rho_{j}-\lambda \rho_{i}}{2+2 \lambda}-\rho_{i} \rho_{j}+O\left(\rho^{3}, \lambda \rho^{2}\right),
$$

where we kept only leading order in $\lambda \approx \lambda_{c} \ll 1$ [26] for quadratic terms in $\rho_{i}$. Plugging Eq. (17) in Eq. (15) with $d \rho_{i} / d t=0$, we obtain

$$
\rho_{i}=\frac{\lambda \sum_{i} B_{i j}(\lambda) \rho_{j}}{1+\lambda \sum_{i} B_{i j}(\lambda) \rho_{j}},
$$

where

$$
B_{i j}=\frac{2+\lambda}{2 \lambda+2} \frac{A_{i j}}{1+\frac{\lambda^{2} k_{i}}{2 \lambda+2}} \simeq \frac{A_{i j}}{1+\frac{\lambda^{2} k_{i}}{2}}
$$

is an effective weighted adjacency matrix. The last passage in Eq. (19) assumes $\lambda \ll 1$.

Equation (18) has exactly the same form of the stationary $\rho_{i}$ in Eq. (11), obtained for QMF theory, replacing $A_{i j}$ by $B_{i j}$. Therefore, all the spectral analysis described in Sec. II C 2 found for QMF theory can be extended to the PQMF case with the replacement of spectral properties of $A_{i j}$ by those of $B_{i j}$. For example, the epidemic threshold is given by

$$
\lambda_{\mathrm{c}}^{\mathrm{PQMF}} \Omega^{(1)}\left(\lambda_{\mathrm{c}}^{\mathrm{PQMF}}\right)=1,
$$

where $\Omega^{(1)}$ is the largest eigenvalue of $B_{i j}$. One can check that this result is exactly the same presented in Ref. [26] expressed in a different way. For $\lambda \gtrsim \lambda_{\mathrm{c}}^{\mathrm{PQMF}}$ we have that $\rho_{i} \sim w_{i}^{(1)}$, where $\left\{w_{i}^{(1)}\right\}$ is the PEV of $B_{i j}\left(\lambda_{\mathrm{c}}^{\mathrm{PQMF}}\right)$ and $\rho \simeq b_{1}\left(\lambda \Omega^{(1)}\left(\lambda_{\mathrm{c}}^{\mathrm{PQMF}}\right)-1\right)$, where $b_{1}(N)$ has the same form as Eq. (13) replacing $v_{i}$ by $w_{i}$. Thus, the IPR of $\left\{w_{i}^{(1)}\right\}$, denoted by $Y_{4}\left[B_{i j}\right]$, allows us to quantify the localization in the PQMF theory.
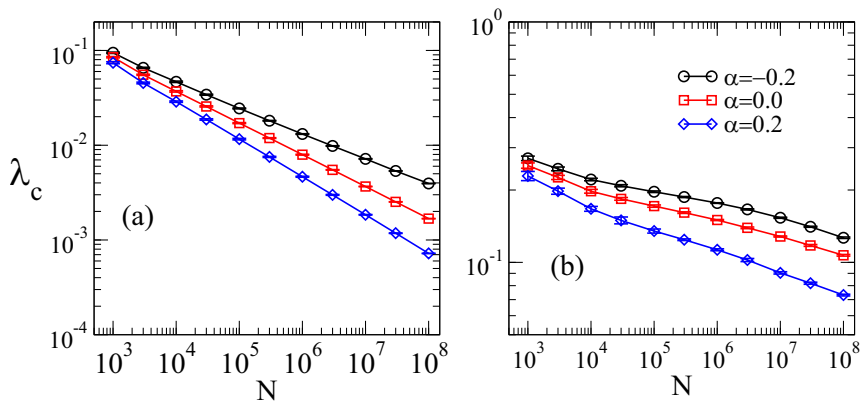

FIG. 2. Threshold as a function of the network size for (a) $\gamma=$ 2.3 and (b) $\gamma=3.5$ and different values of $\alpha$. The lower cutoff is $k_{\min }=3$ for all curves, while the upper cutoff is $k_{\max }=2 \sqrt{N}$ for $\gamma<$ 3 and $k_{\max } \sim N^{1 / \gamma}$ for $\gamma>3$. Curves are averages over ten networks; error bars are smaller than symbols.

\section{RESULTS}

\section{A. Accuracy of theoretical estimates for the epidemic threshold}

Figure 2 shows the dependence of the epidemic threshold as a function of the network size obtained in simulations with different values of $\gamma$ and $\alpha$. We concentrate for the moment on two values of $\gamma$, representative of the cases $\gamma<5 / 2$ and $\gamma>$ 3 , for which the physical mechanisms underlying the epidemic transition are clear [47]. Later we will discuss the case $5 / 2<$ $\gamma<3$, whose interpretation is hampered by extremely long crossover phenomena in the spectral properties. As we can see from this figure, all thresholds vanish as $N$ diverges, regardless of the correlation level $\alpha$ and heterogeneity $\gamma$. Compared to the uncorrelated case, assortative networks $(\alpha>0)$ have a smaller threshold, while the threshold is larger for $\alpha<0$, i.e., disassortative mixing, in agreement with the behavior of the LEV of the adjacency matrix [14,23]. In the case $\gamma>3$, this phenomenology can be qualitatively explained by considering the mechanism of long-range mutual reinfection of hubs $[25,46,48]$, which triggers the epidemic transition. According to this mechanism, the subgraph consisting of the hub plus its nearest neighbors can sustain in isolation an active state for times long enough to permit the activation of other hubs, even if they are not directly connected. This mechanism is at work independently of degree correlations, as long as distances among hubs increase slowly enough with network size. In assortative networks, communication among hubs is enhanced since they have higher probability to be closer; for disassortative topology the converse is true and larger values of $\lambda$ are needed to trigger the transition.

The accuracy of HMF theory is tested with respect to simulations in Fig. 3. For $\gamma=2.3$, we see a non-negligible asymptotic discrepancy between HMF theory and simulations in the case of correlated networks. Interestingly, the HMF theory appears to overestimate the threshold for disassortative networks, while it underestimates it for assortative ones. For larger values of $\gamma$ the discrepancy is conspicuous and the epidemic threshold is significantly overestimated, as can be seen in the insets of Fig. 3.

Comparisons between QMF and PQMF theories and simulations are shown in Figs. 4(a) and 5(a) in the range of network size $10^{3} \leqslant N \leqslant 10^{8}$. For $\gamma=2.3$, both $\mathrm{QMF}$ and PQMF 


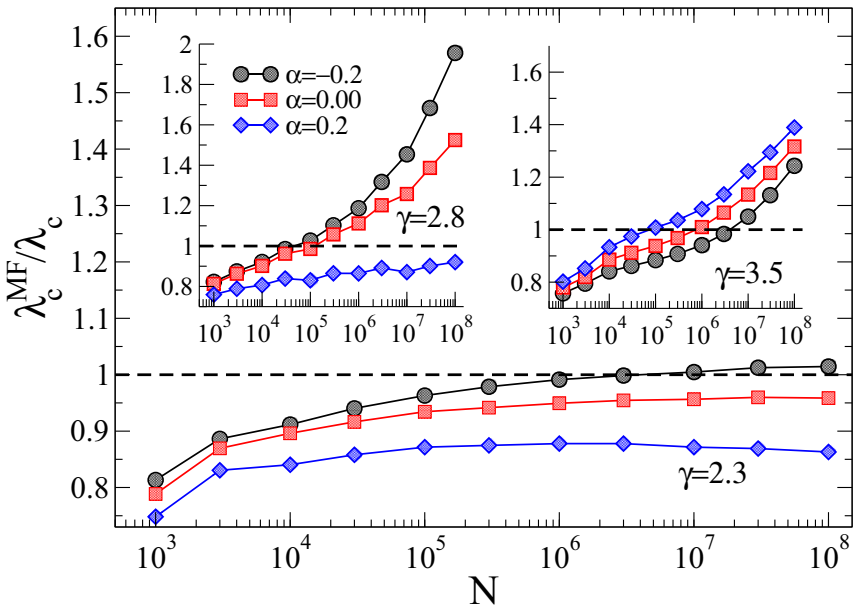

FIG. 3. Ratio between thresholds of HMF theories $\left(\lambda_{c}^{\mathrm{MF}}\right)$ and simulations $\left(\lambda_{c}\right)$ as a function of the network size for different values of $\gamma$ and $\alpha$. The main panel and the right and left insets correspond to $\gamma=2.3,2.8$, and 3.5 respectively. An upper cutoff $k_{\max }=2 \sqrt{N}$ is considered for $\gamma<3$, while for $\gamma=3.5, k_{\max } \sim N^{1 / \gamma}$. Averages correspond to ten network realizations and error bars are smaller than symbols.

theories appear to converge asymptotically to the epidemic threshold observed in simulations. The PQMF theory displays a faster convergence than the QMF theory, this effect being enhanced for smaller values of $\alpha$. For $\gamma=3.5$, the predictions of PQMF and QMF theories succeed, qualitatively, in predicting that the threshold approaches zero in the thermodynamic limit even in the presence of correlations. However, the theoretical
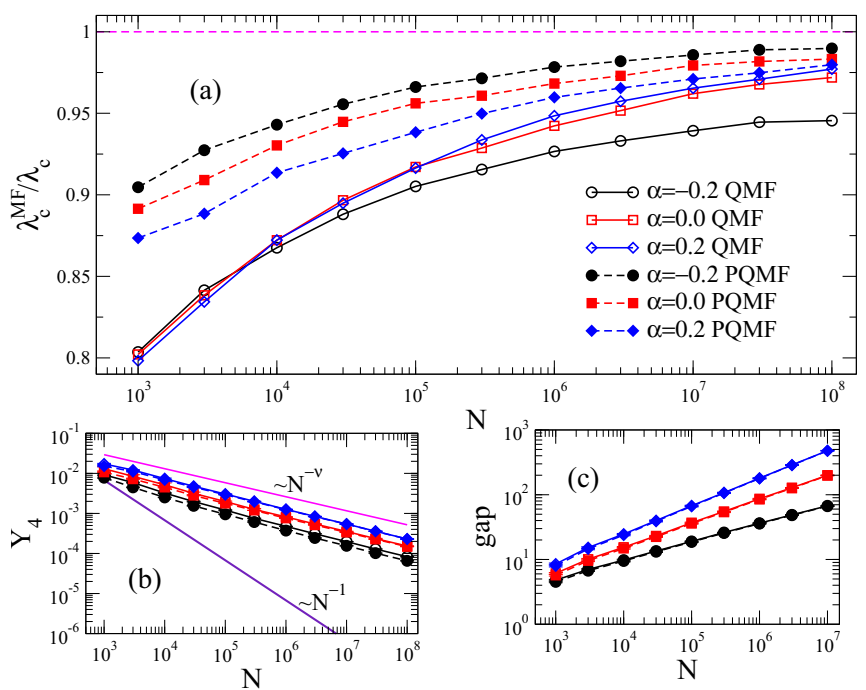

FIG. 4. (a) Comparison of the QMF and PQMF mean-field theories, (b) IPR, and (c) spectral gap of $A_{i j}$ and $B_{i j}$ against size for $\gamma=$ 2.3 and different values of $\alpha$. Averages correspond to ten network realizations. In (b), solid lines are power-law decays $Y_{4} \sim N^{-v}$, with $v=(3-\gamma) / 2$ and $Y_{4} \sim N^{-1}$ corresponding to localization in the maximum $K$-core and finite set of vertices, respectively. Solid lines and open symbols correspond to the QMF theory and $A_{i j}$ analysis, while dashed lines and closed symbols correspond to the PQMF theory and critical $B_{i j}$.

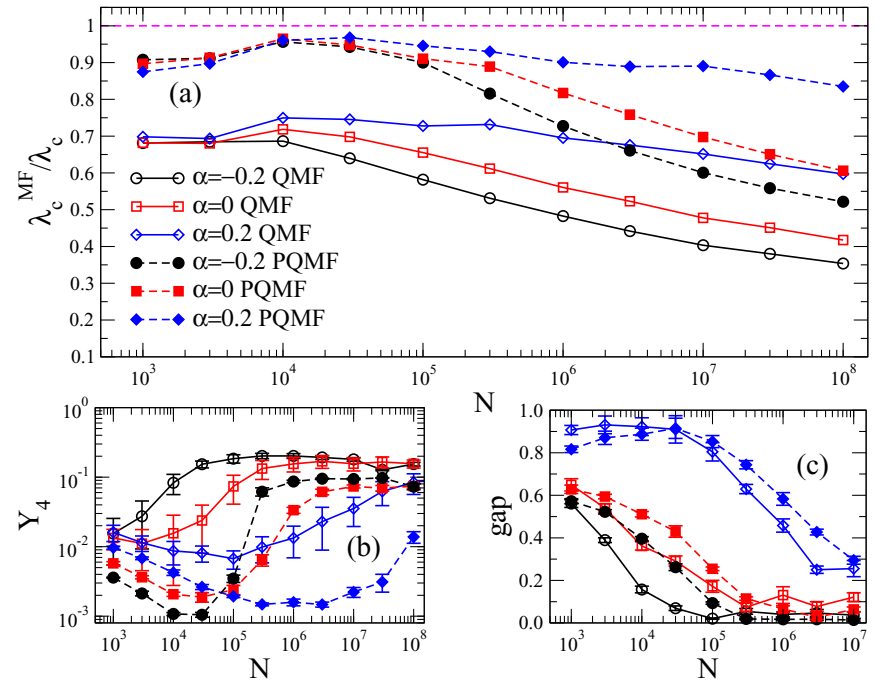

FIG. 5. (a) Comparison of the QMF and PQMF theories, (b) IPR, and (c) spectral gap of $A_{i j}$ and $B_{i j}$ against size for $\gamma=3.5$ and different values of $\alpha$, using an upper cutoff $k_{\max } \sim N^{1 / \gamma}$. Averages correspond to ten network realizations. Solid lines and open symbols correspond to the QMF theory and $A_{i j}$ analysis, while dashed lines and closed symbols correspond to the PQMF theory and critical $B_{i j}$.

threshold estimates depart from simulation results leading to decreasing ratios $\lambda_{\mathrm{c}}^{\mathrm{MF}} / \lambda_{\mathrm{c}}$ in the large network limit. We expect this ratio to decrease asymptotically as $1 / \ln \left(k_{\max }\right)$ [46], in agreement with recent rigorous results [49]. Again, PQMF theory performs better than QMF theory. In this case, the improvement of PQMF over QMF theory grows with $\alpha$.

\section{B. Relation to spectral properties}

What is the origin of the discrepancies between theoretical predictions and numerical results observed in Sec. III A? In this section we investigate which spectral feature is correlated with the performance of the theoretical approaches. We consider both QMF and PQMF theories, testing their accuracy against the spectral properties of adjacency matrices $A_{i j}$ and $B_{i j}$, respectively.

Let us consider first the case $\gamma=3.5$. The real threshold is not the QMF one because the PEV is localized. As pointed out in Ref. [23], this in principle implies that the actual threshold coincides with the inverse of the largest eigenvalue corresponding to a delocalized PEV, coinciding with the HMF threshold $\lambda_{c}^{\mathrm{HMF}}=\langle k\rangle /\left\langle k^{2}\right\rangle$. Actually, however, the QMF approach neglects dynamical correlations, which have the effect of allowing mutual reinfection events among different hubs in the network. In this way an endemic global state can be established due to the long-range interactions among localized states [46] setting the actual threshold to an intermediate value: $\lambda_{c}^{\mathrm{QMF}}<\lambda_{c}<\lambda_{c}^{\mathrm{HMF}}$. With this picture in mind, we can predict that, if the localization is stronger (higher values of the IPR $Y_{4}$ ), the actual threshold will be farther from $\lambda_{c}^{\mathrm{QMF}}$ and thus the performance (accuracy) of the QMF approach will be reduced.

We plot the dependence of $Y_{4}$ on the system size $N$ for $\gamma=3.5$ in Fig. 5(b): The IPR of $A_{i j}$ converges to a finite value in the thermodynamic limit, irrespective of the 
correlation degree, representing a PEV localized on a finite set of vertices [23,43]. The saturation with size occurs earlier for disassortative correlations and later for assortative, compared to the uncorrelated case. In general, for a given size $N, Y_{4}$ is larger for smaller $\alpha$. As expected, a better QMF performance occurs for smaller $Y_{4}$.

The IPR analysis for the PQMF theory, involving $B_{i j}$, has a qualitatively similar behavior to QMF theory, but presents lower values for the IPR. Hence, the PQMF steady-state solution is less localized than that of the QMF theory. Correspondingly, the $\mathrm{PQMF}$ performance is better than the QMF performance. We also calculate, in Fig. 5(c), the dependence of the spectral gap on the system size, for both the adjacency matrix $A_{i j}$ (involved in QMF theory) and $B_{i j}$ (in PQMF theory). The spectral gap is defined as the difference $\Lambda^{(1)}-\Lambda^{(2)}$ between the largest and second largest positive eigenvalues of the adjacency matrices. The gap of the adjacency matrix $A_{i j}$ is small and it decreases as $N$ grows, as predicted by Ref. [50]. The gap is smaller for smaller $\alpha$. The dependence of the spectral gap of $B_{i j}$ on size is qualitatively similar to the gap of $A_{i j}$.

Notice that, while the amplitude of the spectral gap matters for the validity of the QMF prediction for the prevalence above the critical point [Eq. (12)], it does not play any role in the determination of $\lambda_{\mathrm{c}}^{\mathrm{QMF}}$. Therefore, there is no conceptual reason for expecting a correlation between QMF performance and spectral gap size. We find numerically such a correlation in Fig. 5, but we cannot attribute a causal meaning to it.

Let us consider now $\gamma=2.3$. In this case the physical mechanism underlying the epidemic transition is different, as it does not involve the interaction between distant hubs but rather the extension of activity from the maximum $K$-core to the rest of the network. The connection between QMF theory performance and localization is not easily predictable.

As shown in Fig. 4(b), the IPR for $\gamma=2.3$ follows a power law $Y_{4} \sim N^{-v}$, with $v \approx(3-\gamma) / 2$, which corresponds to the IPR localized in the maximum $K$-core of the network [43]. Correlations leave the scaling exponent unchanged, altering only the prefactor: The smaller the $\alpha$, the smaller the IPR. This means that the PEV is still localized on a subextensive fraction of nodes. However, since $Y_{4}$ increases with $\alpha$, the PEV is more localized for positive $\alpha$ than for negative. The same is true for the matrix $B_{i j}$ of the PQMF theory. Interestingly, the effect on the performance of the theoretical approaches is opposite. The QMF theory works better for larger $Y_{4}$ and the PQMF theory works better for smaller $Y_{4}$. We have no simple interpretation for this result.

Figure 4(c) shows the spectral gap for the WPCM networks with $\gamma=2.3$. In this case the gap increases with network size and it is smaller for smaller $\alpha$. This is true also for the spectral gap of the PQMF theory. Finally, let us observe that there is almost no difference between the spectral properties of $A_{i j}$ and $B_{i j}$ for $\gamma=2.3$. This is indeed not surprising for $\alpha=0$ since the term $\lambda^{2} k_{i}$ in the denominator of Eq. (19) is asymptotically negligible, because $\lambda_{c}^{2} k_{\max } \sim k_{\max }^{2 \gamma-5} \rightarrow 0$ as $N \rightarrow \infty$ for $\gamma<$ $5 / 2$.

\section{Intermediate case $2.5<\gamma<3$}

As for the other values of $\gamma$, in this range the vanishing of the threshold with $N$ is observed regardless of the correlation pattern. The localization phenomenon of the PEV in the case

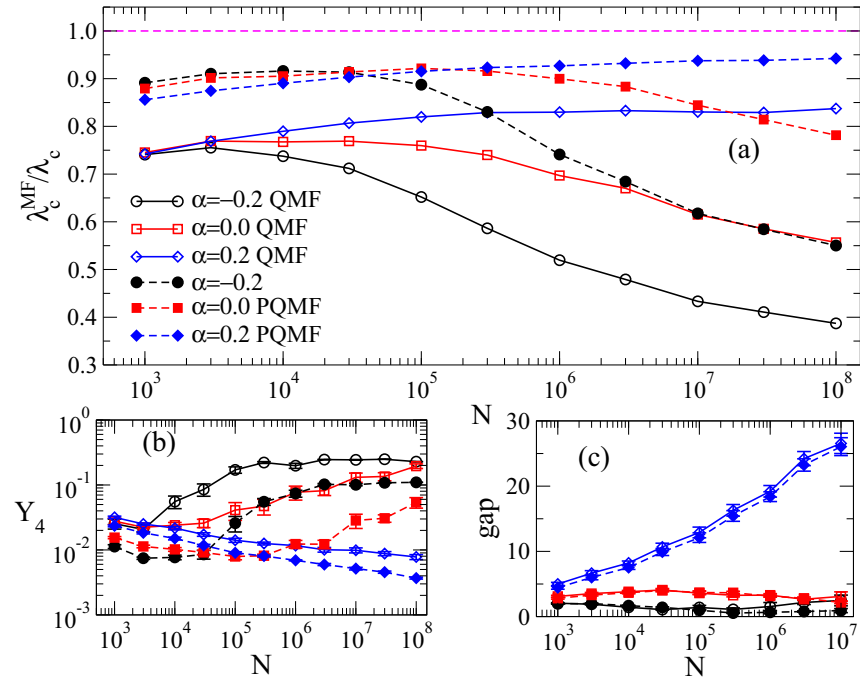

FIG. 6. (a) Comparison of the QMF and PQMF theories, (b) IPR, and (c) spectral gap of $A_{i j}$ and $B_{i j}$ against size for $\gamma=2.8$ and different values of $\alpha$, using an upper cutoff $k_{\max }=2 \sqrt{N}$. Averages correspond to ten network realizations. Solid lines and open symbols correspond to the QMF theory and $A_{i j}$ analysis, while dashed lines and closed symbols correspond to the PQMF theory and critical $B_{i j}$.

$5 / 2<\gamma<3$ is asymptotically analogous to the case $\gamma>3$. However, very strong crossover effects are observed in this case, because of the presence of a localization process on the maximum $K$-core (as for $\gamma<5 / 2$ ) competing with the localization around the hub [43]. As a consequence, already in the uncorrelated case, the PEV gets strongly localized around the largest hub only for very large values of $N$. Correlations further complicate the picture: Figure 6(b) shows that disassortative correlations accelerate the convergence to the final localized state. For $\alpha>0$ instead, $Y_{4}$ is a decreasing function of $N$. The upward bend of the curve hints at an incipient crossover, but one cannot exclude that the asymptotic behavior is different for $\alpha>0$. A similar pattern is observed for the case of the spectral gap [Fig. 6(c)].

With regard to the performance of the theoretical approaches, for negative or zero correlations the scenario perfectly matches what happens for $\gamma>3$ : All theories somehow fail in capturing the way the threshold vanishes, with the PQMF theory being less inaccurate than the others. In the case $\alpha=0.2$ numerical results seem to suggest that both theories describe quite well how the threshold changes with the system size. However, the large crossover effects mentioned above do not allow one to draw any firm conclusion.

We can summarize our findings by stating that the performance in predicting the behavior of epidemic threshold of the QMF and PQMF theories on WPCM networks is correlated with the size of the spectral gap and the IPR of the PEV of the respective $A_{i j}$ and $B_{i j}$ matrices that rule the prevalence near the transition point. A large spectral gap or a low IPR leads to a good performance of the mean-field theories, while the converse, small gap or large IPR, leads to deviations from the theoretical predictions. The QMF theory seems to be more correlated with the spectral gap, while the PQMF theory is with the IPR, at least in the regime where the gap is significant and the theories are accurate. 

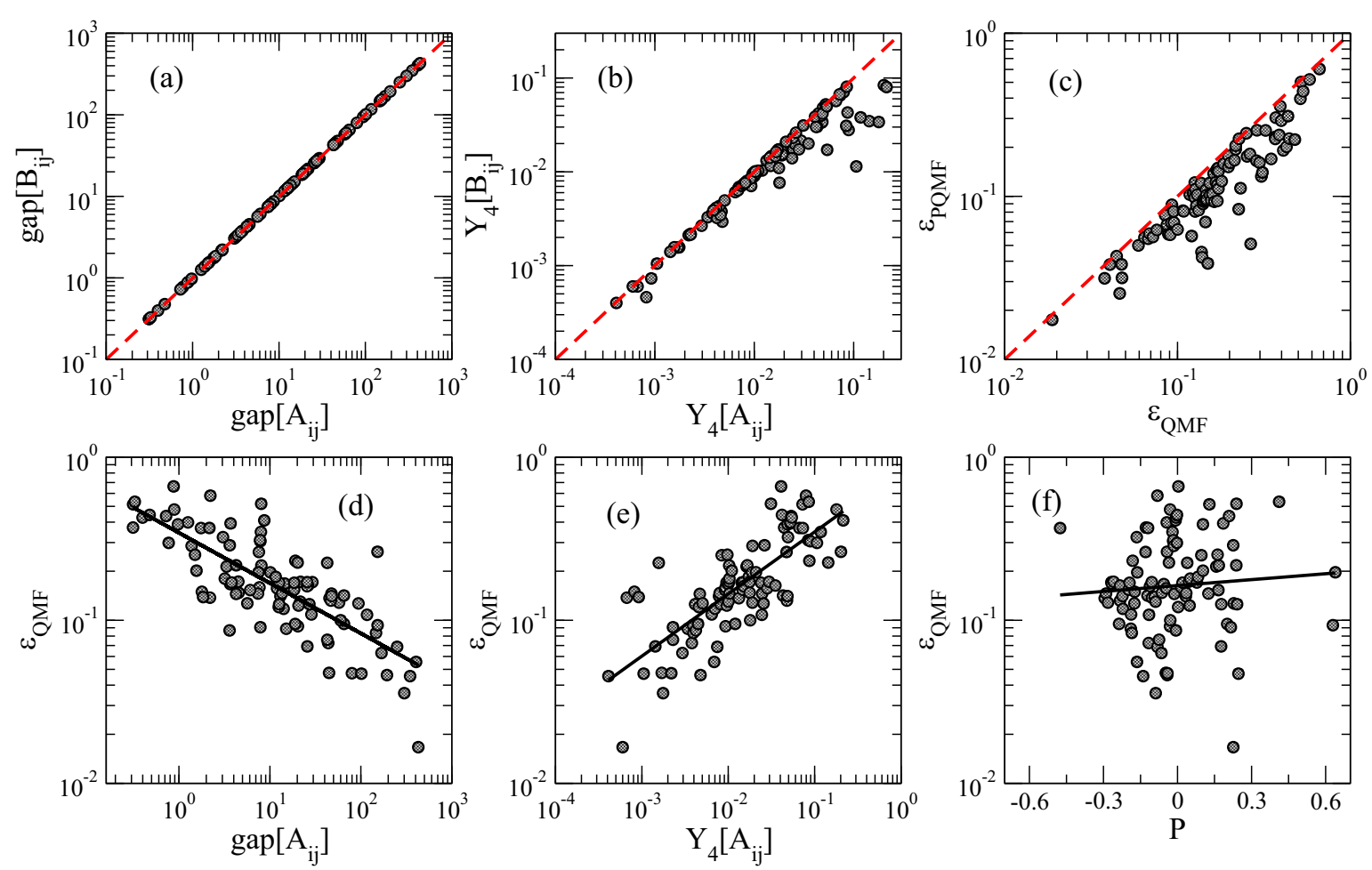

FIG. 7. Scatter plots for a set of 99 real networks (see Appendix). Each point corresponds to a single network. (a) Spectral gap and (b) IPR of the matrix $B_{i j}$ plotted versus the corresponding values for the matrix $A_{i j}$. (c) Relative errors of QMF and PQMF theoretical predictions with respect to the simulation, defined by Eq. (21). Dashed red lines denote the diagonal. Relative errors of the QMF theory are plotted vs (d) the spectral gap, (e) the IPR, and (f) the Pearson coefficient.

\section{Real networks}

We extend our analysis to a set of 99 real-world networks encompassing a broad range of origins, sizes, and topological features (see the Appendix). The spectral gap and IPR of matrices $A_{i j}$ and $B_{i j}$ are compared in the scatter plots shown in Figs. 7(a) and 7(b). We see that the spectral gap is almost the same for both adjacency matrices, while the IPR extracted from $B_{i j}$ is smaller than the one extracted from $A_{i j}$, in particular in the range of large IPR values. This shows that the PQMF matrices $B_{i j}$ are less localized than the matrix $A_{i j}$, relevant for QMF theory. The relative errors between the QMF or PQMF theory and simulations, defined as

$$
\varepsilon=\frac{\lambda_{c}-\lambda_{c}^{\mathrm{MF}}}{\lambda_{c}}
$$

are compared in the scatter plot shown in Fig. 7(c). As in the case of random networks, the PQMF theory outperforms the QMF theory for all networks investigated.

On this set of networks we test the relation observed for synthetic correlated networks, connecting qualitatively the accuracy of QMF and PQMF threshold predictions with the properties of the adjacency matrices (spectral gap and IPR), respectively, and with the Pearson coefficient $P$, measuring network topological correlations. Here $P$ is defined as [2]

$$
P=\frac{\sum_{i j}\left(A_{i j}-\frac{k_{i} k_{j}}{N\langle k\rangle}\right) k_{i} k_{j}}{\sum_{i j}\left(k_{i} \delta_{i j}-\frac{k_{i} k_{j}}{N\langle k\rangle}\right) k_{i} k_{j}} .
$$

The Pearson coefficient lies in the interval $-1<P<1$, being negative for disassortative, null for uncorrelated, and positive for assortative networks. The analyses for QMF theory are shown in the scatter plots of the relative error $\varepsilon$ against the corresponding topological properties in Figs. 7(d) and 7(e). Qualitatively similar patterns obtained for PQMF theory are not shown. We can see that in real networks, the correlation between the performance of the theoretical prediction and the spectral gap is on average the same as that observed for the WPCM: A larger spectral gap is associated with a higher accuracy. The inverse correlation with the IPR is again preserved: A smaller $Y_{4}$ corresponds to a more accurate prediction. We do not find a significant correlation with the Pearson coefficient. Statistical analyses were performed using the correlation coefficients obtained from either power law, in the case of the spectral gap and IPR, or from exponential, in the case of the Pearson coefficient, regressions of the scatter plots. We obtain strong statistical correlations with $|r| \gtrsim$ $0.70\left(p<10^{-5}\right)$ for both QMF and PQMF theories using either the IPR or spectral gap of the corresponding matrices. Values $r \lesssim 0.2(p>0.05)$ for correlation with the Pearson coefficient of the network confirm no significant statistical correlations.

\section{E. Epidemic prevalence near the epidemic threshold}

Figure 2 shows that the QMF prediction for the epidemic threshold tends to the same limit of numerical simulations for both uncorrelated and correlated networks for $\gamma=2.3$. This observation naturally leads to one wonder whether the QMF theory is asymptotically an exact description for SIS dynamics 


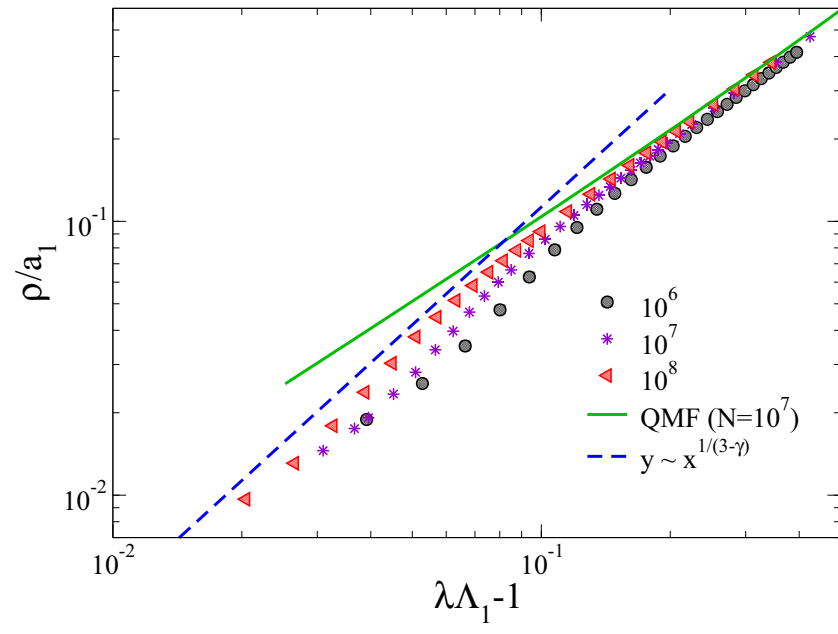

FIG. 8. Rescaled average density as a function of the distance from the epidemic threshold. Quasistationary simulations for different sizes are indicated in the legend. The solid line is a numerical integration of the QMF theory [Eq. (9)] for $N=10^{7}$, while the dashed line is a power law with exponent predicted analytically in Ref. [51]. We used uncorrelated networks $(\alpha=0)$ with degree exponent $\gamma=2.3$ and $k_{\max }=2 \sqrt{N}$.

on random networks with $\gamma<5 / 2$. In order to answer this question we test the exactness of the other prediction of the QMF theory, Eq. (12), stating that the fraction of infected individuals decays to zero linearly as the threshold is approached from above. Numerical results, for the case of uncorrelated networks $\alpha=0$ are shown in Fig. 8, where the density and the infection rates are rescaled to conform to Eq. (12). We can clearly see the existence of two scaling regimes. For $\lambda \Lambda_{1}-$ $1 \ll 1$ the density scales with an exponent larger than the prediction $\beta^{(\mathrm{QMF})}=1$. The observed exponent is consistent with the exact result of Ref. [51], $\beta=1 /(3-\gamma)$, which is also (probably accidentally) the value predicted by HMF theory [52]. This exponent is observed in a regime very close to the transition, where the system is kept asymptotically active only by virtue of the quasistationary method. We performed a nonperturbative analysis by integrating the QMF equations using a fourth-order Runge-Kutta method for $\lambda>\frac{1}{\Lambda^{(1)}}$ for $N=10^{7}$. A comparison with simulation results confirms that the QMF theory correctly predicts the linear behavior of the prevalence $\rho$ around the epidemic transition, but only sufficiently far from it. In the immediate neighborhood of the threshold the decay is more rapid.

\section{CONCLUSION}

The determination of the epidemic threshold in models of disease spreading in complex topologies is a nontrivial problem in network science. Several theoretical approaches have been proposed, applying approximations with different levels of stringency, that provide contrasting predictions on the epidemic threshold. Among the main theoretical approaches at the mean-field level we can consider, in decreasing order of approximation, the heterogeneous mean-field theory, neglecting dynamical correlations and the actual pattern of connections in the network (preserving only its statistical properties); the quenched mean-field theory, also neglecting dynamical correlations but keeping the network structure; and the pair quenched mean-field theory, which incorporates dynamical correlations between pairs of connected nodes. In this paper we have presented a comparison of the predictions of these three approximate theories for the case of the susceptible-infected-susceptible epidemic model, focusing on the case of networks with a power-law degree distribution and degree correlations, representative of many real network systems.

Comparing the predictions with actual stochastic simulations of the SIS process, we observed that, independently of the degree of correlations, the predictions of PQMF theory are more accurate than those of QMF theory, while both outperform HMF theory, which fails to predict the vanishing threshold observed for a degree exponent $\gamma>3$. While overall PQMF theory is more accurate than QMF theory, the two approximations show different levels of accuracy when compared in networks with different levels of correlations. Thus, for the case of synthetic networks generated with the WeberPorto algorithm [35], we observed that, for fixed network size and degree heterogeneity, QMF predictions are more accurate in assortative networks than in disassortative ones. On the other hand, PQMF theory is increasingly accurate in the presence of disassortative correlations for small degree exponent, while it is more accurate when correlations are assortative if the degree exponent is large.

We proposed a criterion for the accuracy of the QMF and PQMF approaches based on the spectral properties of the networks. We observed that the accuracy is positively correlated with the amplitude of the spectral gap of the adjacency matrix and is inversely related to the degree of localization of the principal eigenvalue, as measured by the inverse participation ratio. This general observation was corroborated by the analysis of a large set of real correlated networks, covering a wide range of sizes and topological features.

Additionally, we investigated the behavior of the order parameter of the transition, measured in terms of the prevalence or density of infected nodes in the steady state, for $\gamma<5 / 2$. We observe that, in uncorrelated synthetic networks, the linear decay (critical exponent $\beta=1$ ) predicted by QMF theory is observed in stochastic simulations not very close to the transition. When fluctuation effects become more important, i.e., very close to the transition, the observed exponent $\beta$ crosses over to the value $\beta=1 /(3-\gamma)$, in agreement with rigorous mathematical results [51].

\section{ACKNOWLEDGMENTS}

This work was partially supported by the Brazilian agencies CAPES, CNPq, and FAPEMIG. D.H.S. and S.C.F. are grateful for support from the CAPES program Ciência sem Fronteiras, under Project No. 88881.030375/2013-01. R.P.-S. and C.C. acknowledge financial support from the Spanish MINECO, under Project No. FIS2016-76830-C2-1-P. R.P.-S. acknowledges additional financial support from ICREA Academia, funded by the Generalitat de Catalunya. This study was financed in part by the Coordenação de Aperfeiçoamento de Pessoal de Nível Superior (Brazil), Grant No. 001. 
TABLE I. Properties of the set with 99 networks of distinct types. We show the network size $N$, the average degree $\langle k\rangle$, the Pearson coefficient $P$, the IPRs of both $A_{i j}\left(\mathrm{IPR}_{\mathrm{A}}\right)$ and critical $B_{i j}\left(\mathrm{IPR}_{\mathrm{B}}\right)$ matrices, the spectral gap of $A_{i j}\left(\Delta \Lambda_{\mathrm{A}}^{1,2}\right)$, and the thresholds of simulations $\left(\lambda_{c}\right)$, QMF theory $\left(\lambda_{c}^{\mathrm{QMF}}\right)$, and PQMF theory $\left(\lambda_{c}^{\mathrm{PQMF}}\right)$.

\begin{tabular}{|c|c|c|c|c|c|c|c|c|c|}
\hline Network & $N$ & $\langle k\rangle$ & $P$ & $\mathrm{IPR}_{\mathrm{A}}$ & $\mathrm{IPR}_{\mathrm{B}}$ & $\Delta \Lambda_{\mathrm{A}}^{1,2}$ & $\lambda_{c}$ & $\lambda_{c}^{\mathrm{QMF}}$ & $\lambda_{c}^{\mathrm{PQMF}}$ \\
\hline Karate club & 34 & 4.59 & -0.476 & 0.0730 & 0.0649 & 1.75 & 0.235 & 0.149 & 0.181 \\
\hline Radoslaw Email & 167 & 38.9 & -0.295 & 0.0133 & 0.0132 & 45.2 & 0.0191 & 0.0165 & 0.0168 \\
\hline Spanish B & 12643 & 8.70 & -0.290 & 0.0246 & 0.0174 & 47.5 & 0.0105 & 0.00897 & 0.00951 \\
\hline Spanish A & 11558 & 7.45 & -0.282 & 0.0190 & 0.0150 & 57.5 & 0.0113 & 0.00985 & 0.0103 \\
\hline US Air Transportation & 500 & 11.9 & -0.268 & 0.0176 & 0.0173 & 29.3 & 0.0251 & 0.0208 & 0.0214 \\
\hline Little Rock Lake & 183 & 26.6 & -0.266 & 0.0148 & 0.0145 & 14.6 & 0.0291 & 0.0242 & 0.0249 \\
\hline Japanese & 2698 & 5.93 & -0.259 & 0.0296 & 0.0214 & 21.7 & 0.0281 & 0.0233 & 0.0250 \\
\hline English & 7377 & 12.0 & -0.237 & 0.0120 & 0.0103 & 65.3 & 0.0101 & 0.00914 & 0.0094 \\
\hline French & 8308 & 5.74 & -0.233 & 0.0351 & 0.0200 & 26.3 & 0.0197 & 0.0165 & 0.0179 \\
\hline Jung & 6120 & 16.4 & -0.233 & 0.0478 & 0.0335 & 46.9 & 0.00810 & 0.00703 & 0.00743 \\
\hline JDK & 6434 & 16.7 & -0.223 & 0.0484 & 0.0341 & 47.6 & 0.00810 & 0.00696 & 0.00737 \\
\hline Political blogs & 1222 & 27.4 & -0.221 & 0.00701 & 0.00687 & 14.1 & 0.0153 & 0.0135 & 0.0137 \\
\hline Internet & 22963 & 4.22 & -0.198 & 0.0146 & 0.0116 & 18.4 & 0.0165 & 0.0140 & 0.0148 \\
\hline AS Caida & 26475 & 4.03 & -0.195 & 0.0240 & 0.0140 & 18.5 & 0.0173 & 0.0144 & 0.0157 \\
\hline EU email & 224832 & 3.02 & -0.189 & 0.00340 & 0.00328 & 15.2 & 0.0107 & 0.00975 & 0.0101 \\
\hline UC Irvine & 1893 & 14.6 & -0.188 & 0.00643 & 0.00608 & 28.6 & 0.0233 & 0.0208 & 0.0214 \\
\hline Linux, mailing list & 24567 & 12.9 & -0.185 & 0.00395 & 0.00386 & 147 & 0.00490 & 0.00448 & 0.00452 \\
\hline AS Oregon & 6474 & 3.88 & -0.182 & 0.0868 & 0.0429 & 19.1 & 0.0281 & 0.0216 & 0.0249 \\
\hline Linux, soft. & 30817 & 13.8 & -0.175 & 0.0256 & 0.0197 & 94.1 & 0.00670 & 0.00585 & 0.00616 \\
\hline Gnutella, 25 August 2002 & 22663 & 4.83 & -0.173 & 0.000815 & 0.000464 & 1.79 & 0.108 & 0.0916 & 0.104 \\
\hline Les Miserables & 77 & 6.60 & -0.165 & 0.0492 & 0.0482 & 3.05 & 0.123 & 0.0833 & 0.0919 \\
\hline Petster-cats & 148826 & 73.2 & -0.164 & 0.00687 & 0.00635 & 405 & 0.000900 & 0.000847 & 0.000855 \\
\hline C. Elegans, neural & 297 & 14.5 & -0.163 & 0.0189 & 0.0176 & 10.1 & 0.0511 & 0.0410 & 0.0434 \\
\hline Libimseti & 220970 & 156 & -0.139 & 0.000406 & 0.000398 & 348 & 0.00110 & 0.00106 & 0.00106 \\
\hline David Copperfield & 112 & 7.59 & -0.129 & 0.0473 & 0.0397 & 7.57 & 0.103 & 0.0760 & 0.0844 \\
\hline Political books & 105 & 8.40 & -0.128 & 0.0444 & 0.0419 & 0.313 & 0.133 & 0.0838 & 0.0927 \\
\hline Google & 15763 & 18.9 & -0.122 & 0.0430 & 0.0303 & 65.1 & 0.00670 & 0.00575 & 0.00608 \\
\hline Social 3 & 32 & 5.00 & -0.119 & 0.0665 & 0.0568 & 2.16 & 0.265 & 0.167 & 0.205 \\
\hline Euron & 33696 & 10.7 & -0.116 & 0.00379 & 0.00361 & 43.9 & 0.00910 & 0.00844 & 0.00859 \\
\hline Web Stanford & 255265 & 15.2 & -0.116 & 0.0245 & 0.0230 & 117 & 0.00250 & 0.00223 & 0.0023 \\
\hline Bay Wet & 128 & 32.4 & -0.112 & 0.0151 & 0.0147 & 25.6 & 0.0301 & 0.0252 & 0.0259 \\
\hline Bay Dry & 128 & 32.9 & -0.104 & 0.0148 & 0.0145 & 25.7 & 0.0301 & 0.0249 & 0.0256 \\
\hline Gnutella, 30 August 2002 & 36646 & 4.82 & -0.104 & 0.000672 & 0.000604 & 2.19 & 0.0897 & 0.0773 & 0.0856 \\
\hline Gnutella, 31 August 2002 & 62561 & 4.73 & -0.0927 & 0.000921 & 0.000731 & 1.85 & 0.0881 & 0.0759 & 0.0844 \\
\hline Petster-hamster & 1788 & 14.0 & -0.0889 & 0.0100 & 0.00938 & 21.6 & 0.0249 & 0.0217 & 0.0223 \\
\hline Petster-dogs & 426485 & 40.1 & -0.0884 & 0.00176 & 0.00157 & 300 & 0.00140 & 0.00135 & 0.00136 \\
\hline Network Science & 379 & 4.82 & -0.0817 & 0.0794 & 0.0705 & 2.21 & 0.230 & 0.0964 & 0.110 \\
\hline AS Skitter & 1694616 & 13.1 & -0.0814 & 0.00746 & 0.00709 & 251 & 0.00160 & 0.00149 & 0.00151 \\
\hline Slashdot zoo & 79116 & 11.8 & -0.0746 & 0.00229 & 0.00218 & 42.8 & 0.00830 & 0.00767 & 0.00778 \\
\hline Wikipedia, edits & 113123 & 35.8 & -0.0651 & 0.00295 & 0.00266 & 169 & 0.0027 & 0.00253 & 0.00255 \\
\hline CiteSeer & 365154 & 9.43 & -0.0632 & 0.0177 & 0.0110 & 4.54 & 0.0202 & 0.0172 & 0.0183 \\
\hline Cora & 23166 & 7.70 & -0.0553 & 0.0100 & 0.00898 & 3.66 & 0.0381 & 0.0317 & 0.0334 \\
\hline Thesaurus & 23132 & 25.7 & -0.0477 & 0.0017 & 0.00156 & 44.7 & 0.0105 & 0.0100 & 0.0102 \\
\hline DBLP, citations & 12495 & 7.93 & -0.0461 & 0.0282 & 0.0174 & 12.0 & 0.0277 & 0.0234 & 0.0251 \\
\hline Dolphins & 62 & 5.13 & -0.0436 & 0.0526 & 0.0493 & 1.26 & 0.231 & 0.139 & 0.164 \\
\hline DBpedia & 3915921 & 6.42 & -0.0427 & 0.201 & 0.0840 & 153 & 0.00190 & 0.00140 & 0.00180 \\
\hline Wikipedia, pages & 2070367 & 40.9 & -0.0418 & 0.00477 & 0.00294 & 194 & 0.00130 & 0.00124 & 0.00127 \\
\hline Epinions & 75877 & 10.7 & -0.0406 & 0.00219 & 0.00211 & 79.8 & 0.00570 & 0.00543 & 0.00548 \\
\hline Slashdot & 51083 & 4.56 & -0.0347 & 0.144 & 0.0347 & 20.2 & 0.0219 & 0.0170 & 0.0201 \\
\hline Hep-Th, citations & 27400 & 25.7 & -0.0305 & 0.00931 & 0.00705 & 20.3 & 0.00990 & 0.00899 & 0.00922 \\
\hline S 838 & 512 & 3.20 & -0.0300 & 0.179 & 0.0340 & 0.889 & 0.382 & 0.200 & 0.297 \\
\hline Gowalla & 196591 & 9.67 & -0.0293 & 0.0180 & 0.00764 & 60.0 & 0.00650 & 0.00585 & 0.00609 \\
\hline Amazon, 12 March 2003 & 400727 & 11.7 & -0.0203 & 0.118 & 0.0381 & 7.97 & 0.0273 & 0.0178 & 0.0227 \\
\hline Amazon, 6 June 2003 & 403364 & 12.1 & -0.0176 & 0.0891 & 0.0279 & 7.87 & 0.0252 & 0.0175 & 0.0219 \\
\hline Amazon, 5 May 2003 & 410236 & 11.9 & -0.0169 & 0.0843 & 0.0309 & 7.79 & 0.0249 & 0.0172 & 0.0214 \\
\hline Air traffic & 1226 & 3.93 & -0.0152 & 0.0191 & 0.0154 & 1.38 & 0.152 & 0.109 & 0.127 \\
\hline Gnutella, 4 August 2002 & 10876 & 7.35 & -0.0132 & 0.00469 & 0.00377 & 4.26 & 0.0685 & 0.0586 & 0.0637 \\
\hline
\end{tabular}


TABLE I. (Continued.)

\begin{tabular}{|c|c|c|c|c|c|c|c|c|c|}
\hline Network & $N$ & $\langle k\rangle$ & $P$ & $\mathrm{IPR}_{\mathrm{A}}$ & $\mathrm{IPR}_{\mathrm{B}}$ & $\Delta \Lambda_{\mathrm{A}}^{1,2}$ & $\lambda_{c}$ & $\lambda_{c}^{\mathrm{QMF}}$ & $\lambda_{c}^{\mathrm{PQMF}}$ \\
\hline Gnutella, 24 August 2002 & 26498 & 4.93 & -0.00778 & 0.214 & 0.0800 & 8.68 & 0.0865 & 0.0511 & 0.0700 \\
\hline Hep-Ph, citations & 34401 & 24.5 & -0.00644 & 0.00421 & 0.00323 & 3.61 & 0.0143 & 0.0131 & 0.0133 \\
\hline S 420 & 252 & 3.17 & -0.00591 & 0.0542 & 0.0172 & 0.398 & 0.400 & 0.229 & 0.320 \\
\hline Amazon, 2 May 2003 & 262111 & 6.87 & -0.00248 & 0.106 & 0.0114 & 0.771 & 0.0605 & 0.0425 & 0.0508 \\
\hline S 208 & 122 & 3.10 & -0.00201 & 0.0419 & 0.0301 & 0.475 & 0.437 & 0.244 & 0.338 \\
\hline Digg & 29652 & 5.72 & 0.00265 & 0.00457 & 0.00346 & 12.5 & 0.0369 & 0.0324 & 0.0348 \\
\hline US Power grid & 4941 & 2.67 & 0.00346 & 0.0409 & 0.0386 & 0.874 & 0.396 & 0.134 & 0.157 \\
\hline Gnutella, 5 August 2002 & 8842 & 7.20 & 0.0146 & 0.00931 & 0.00855 & 7.68 & 0.0505 & 0.0425 & 0.0453 \\
\hline Jazz & 198 & 27.7 & 0.0202 & 0.0143 & 0.0141 & 12.6 & 0.0301 & 0.0250 & 0.0257 \\
\hline Gnutella, 9 August 2002 & 8104 & 6.42 & 0.0331 & 0.00782 & 0.00737 & 13.1 & 0.0409 & 0.0351 & 0.0370 \\
\hline Gnutella, 8 August 2002 & 6299 & 6.60 & 0.0355 & 0.00795 & 0.00752 & 13.7 & 0.0413 & 0.0352 & 0.0371 \\
\hline LiveJournal & 5189808 & 18.8 & 0.0394 & 0.00157 & 0.00157 & 42.7 & 0.00240 & 0.00186 & 0.00186 \\
\hline High school, 2012 & 180 & 24.7 & 0.0464 & 0.0102 & 0.0101 & 4.49 & 0.0401 & 0.0332 & 0.0344 \\
\hline Open flights & 2905 & 10.8 & 0.0489 & 0.00963 & 0.00942 & 20.6 & 0.0181 & 0.0159 & 0.0162 \\
\hline Gnutella, 6 August 2002 & 8717 & 7.23 & 0.0516 & 0.0103 & 0.00957 & 3.20 & 0.0545 & 0.0447 & 0.0478 \\
\hline URV email & 1133 & 9.62 & 0.0782 & 0.00956 & 0.00865 & 3.78 & 0.0581 & 0.0482 & 0.0512 \\
\hline High school, 2011 & 126 & 27.1 & 0.0829 & 0.0173 & 0.0171 & 11.6 & 0.0361 & 0.0294 & 0.0304 \\
\hline DBLP, collaborations & 1137114 & 8.83 & 0.0964 & 0.00797 & 0.00840 & 0.0594 & 0.0113 & 0.00847 & 0.00855 \\
\hline MathSciNet & 332689 & 4.93 & 0.103 & 0.0110 & 0.0103 & 1.56 & 0.0347 & 0.0277 & 0.0291 \\
\hline Social 1 & 67 & 4.24 & 0.103 & 0.0486 & 0.0418 & 0.975 & 0.292 & 0.179 & 0.223 \\
\hline Cond-Mat, 1993-2003 & 21363 & 8.55 & 0.125 & 0.0103 & 0.00947 & 7.41 & 0.0309 & 0.0264 & 0.0275 \\
\hline Protein 1 & 95 & 4.48 & 0.129 & 0.0723 & 0.0670 & 0.314 & 0.384 & 0.187 & 0.232 \\
\hline Cond-Mat, 1995-1999 & 13861 & 6.44 & 0.157 & 0.0163 & 0.0146 & 3.34 & 0.0509 & 0.0400 & 0.0424 \\
\hline College football & 115 & 10.7 & 0.162 & 0.00977 & 0.00967 & 1.50 & 0.124 & 0.0928 & 0.102 \\
\hline Cond-Mat, 1995-2003 & 27519 & 8.44 & 0.166 & 0.00917 & 0.00847 & 6.09 & 0.0293 & 0.0248 & 0.0258 \\
\hline US Patents & 3764117 & 8.77 & 0.168 & 0.0103 & 0.0100 & 8.05 & 0.0113 & 0.00885 & 0.00899 \\
\hline Facebook links & 63392 & 25.8 & 0.177 & 0.00143 & 0.00140 & 25.8 & 0.00810 & 0.00754 & 0.00762 \\
\hline Cond-Mat, 1995-2005 & 36458 & 9.42 & 0.177 & 0.00814 & 0.00761 & 12.7 & 0.0223 & 0.0195 & 0.0201 \\
\hline Hep-Th, 1995-1999 & 5835 & 4.74 & 0.185 & 0.0523 & 0.0523 & 3.70 & 0.0913 & 0.0554 & 0.0587 \\
\hline AstroPhys, 1993-2003 & 17903 & 22.0 & 0.201 & 0.00447 & 0.00432 & 18.9 & 0.0117 & 0.0106 & 0.0108 \\
\hline Protein 2 & 53 & 4.64 & 0.209 & 0.0536 & 0.0500 & 0.722 & 0.305 & 0.172 & 0.210 \\
\hline Facebook wall & 43953 & 8.30 & 0.216 & 0.00229 & 0.00214 & 7.86 & 0.0277 & 0.0252 & 0.0261 \\
\hline Dublin & 410 & 13.5 & 0.226 & 0.0263 & 0.0261 & 3.62 & 0.0601 & 0.0428 & 0.0448 \\
\hline Actor coll. net. & 374511 & 80.2 & 0.226 & 0.000600 & 0.000599 & 429 & 0.00120 & 0.00118 & 0.00118 \\
\hline Astrophysics & 14845 & 16.1 & 0.228 & 0.00504 & 0.00494 & 5.64 & 0.0155 & 0.0135 & 0.0138 \\
\hline PGP & 10680 & 4.55 & 0.238 & 0.0166 & 0.0163 & 4.25 & 0.0301 & 0.0236 & 0.0243 \\
\hline Hep-Th, 1993-2003 & 8638 & 5.74 & 0.239 & 0.0312 & 0.0312 & 8.03 & 0.0669 & 0.0322 & 0.0333 \\
\hline Reactome & 5973 & 48.8 & 0.241 & 0.00414 & 0.00413 & 27.2 & 0.00550 & 0.00481 & 0.00483 \\
\hline Flickr & 105722 & 43.8 & 0.247 & 0.00105 & 0.00105 & 101 & 0.00170 & 0.00162 & 0.00163 \\
\hline E. coli, transcription & 97 & 4.37 & 0.412 & 0.0854 & 0.0807 & 0.327 & 0.328 & 0.153 & 0.184 \\
\hline Hep-Ph, 1993-2003 & 11204 & 21.0 & 0.630 & 0.00389 & 0.00389 & 153 & 0.00450 & 0.00408 & 0.0041 \\
\hline GR-QC, 1993-2003 & 4158 & 6.46 & 0.639 & 0.0209 & 0.0209 & 7.49 & 0.0273 & 0.0219 & 0.0225 \\
\hline
\end{tabular}

\section{APPENDIX: SUMMARY OF REAL} NETWORK PROPERTIES

We consider 99 real networks with diverse structural properties, based on the lists of Refs. [53,54]. Here we investigate their giant connected components, after symmetrizing all edges (weighted and/or directed) and avoiding multiple and self-connections. The list of networks with some topological properties and epidemic (SIS) parameters is shown in Table I. For detailed information about the original references for all the networks, see Refs. [53,54].
[1] A. Vázquez, A. Flammini, A. Maritan, and A. Vespignani, Modeling of protein interaction networks, Complexus 1, 38 (2002).

[2] M. Newman, Networks: An Introduction (Oxford University Press, Oxford, 2010).

[3] C. Cattuto, W. V. den Broeck, A. Barrat, V. Colizza, J.-F. Pinton, and A. Vespignani, Dynamics of person-to-person interactions from distributed RFID sensor networks, PLoS One 5, e11596 (2010).

[4] A.-L. Barabási and R. Albert, Emergence of scaling in random networks, Science 286, 509 (1999).

[5] R. Albert and A.-L. Barabási, Statistical mechanics of complex networks, Rev. Mod. Phys. 74, 47 (2002). 
[6] R. Pastor-Satorras, A. Vázquez, and A. Vespignani, Dynamical and Correlation Properties of the Internet, Phys. Rev. Lett. 87, 258701 (2001).

[7] M. E. J. Newman, Assortative Mixing in Networks, Phys. Rev. Lett. 89, 208701 (2002).

[8] A. Barrat, M. Barthelemy, and A. Vespignani, Dynamical Processes on Complex Networks (Cambridge University Press, Cambridge, 2008).

[9] R. Pastor-Satorras, C. Castellano, P. Van Mieghem, and A. Vespignani, Epidemic processes in complex networks, Rev. Mod. Phys. 87, 925 (2015)

[10] W. Wang, M. Tang, H. E. Stanley, and L. A. Braunstein, Unification of theoretical approaches for epidemic spreading on complex networks, Rep. Prog. Phys. 80, 036603 (2017).

[11] M. Boguñá, R. Pastor-Satorras, and A. Vespignani, Absence of Epidemic Threshold in Scale-Free Networks with Degree Correlations, Phys. Rev. Lett. 90, 028701 (2003).

[12] V. M. Eguíluz and K. Klemm, Epidemic Threshold in Structured Scale-Free Networks, Phys. Rev. Lett. 89, 108701 (2002).

[13] C. P. Warren, L. M. Sander, and I. M. Sokolov, Geography in a scale-free network model, Phys. Rev. E 66, 056105 (2002).

[14] P. van Mieghem, H. Wang, X. Ge, S. Tang, and F. A. Kuipers, Influence of assortativity and degree-preserving rewiring on the spectra of networks, Eur. Phys. J. B 76, 643 (2010).

[15] A. V. Goltsev, S. N. Dorogovtsev, and J. F. F. Mendes, Percolation on correlated networks, Phys. Rev. E 78, 051105 (2008).

[16] E. Kenah and J. C. Miller, Epidemic percolation networks, epidemic outcomes, and interventions, Interdiscip. Perspect. Infect. Dis. 2011, 543520 (2011).

[17] R. Pastor-Satorras and A. Vespignani, Epidemic Spreading in Scale-Free Networks, Phys. Rev. Lett. 86, 3200 (2001).

[18] A.-L. Barabási and M. Pósfai, Network Science (Cambridge University Press, Cambridge, 2016).

[19] Y. Wang, D. Chakrabarti, C. Wang, and C. Faloutsos, Proceedings of the 22nd International Symposium on Reliable Distributed Systems, Florence, 2003 (IEEE, Piscataway, 2003), pp. 25-34.

[20] D. Chakrabarti, Y. Wang, C. Wang, J. Leskovec, and C. Faloutsos, Epidemic thresholds in real networks, ACM T. Inform. Syst. Se. 10, 1 (2008).

[21] C. Castellano and R. Pastor-Satorras, Thresholds for Epidemic Spreading in Networks, Phys. Rev. Lett. 105, 218701 (2010).

[22] S. C. Ferreira, C. Castellano, and R. Pastor-Satorras, Epidemic thresholds of the susceptible-infected-susceptible model on networks: A comparison of numerical and theoretical results, Phys. Rev. E 86, 041125 (2012).

[23] A. V. Goltsev, S. N. Dorogovtsev, J. G. Oliveira, and J. F. F. Mendes, Localization and Spreading of Diseases in Complex Networks, Phys. Rev. Lett. 109, 128702 (2012).

[24] H. K. Lee, P.-S. Shim, and J. D. Noh, Epidemic threshold of the susceptible-infected-susceptible model on complex networks, Phys. Rev. E 87, 062812 (2013).

[25] M. Boguñá, C. Castellano, and R. Pastor-Satorras, Nature of the Epidemic Threshold for the Susceptible-Infected-Susceptible Dynamics in Networks, Phys. Rev. Lett. 111, 068701 (2013).
[26] A. S. Mata and S. C. Ferreira, Pair quenched mean-field theory for the susceptible-infected-susceptible model on complex networks, Europhys. Lett. 103, 48003 (2013).

[27] A. S. Mata and S. C. Ferreira, Multiple transitions of the susceptible-infected-susceptible epidemic model on complex networks, Phys. Rev. E 91, 012816 (2015).

[28] C. Castellano and R. Pastor-Satorras, Relating Topological Determinants of Complex Networks to their Spectral Properties: Structural and Dynamical Effects, Phys. Rev. X 7, 041024 (2017).

[29] M. Catanzaro, M. Boguñá, and R. Pastor-Satorras, Generation of uncorrelated random scale-free networks, Phys. Rev. E 71, 027103 (2005).

[30] S. Chatterjee and R. Durrett, Contact processes on random graphs with power law degree distributions have critical value 0, Ann. Probab. 37, 2332 (2009).

[31] J. P. Gleeson, High-Accuracy Approximation of BinaryState Dynamics on Networks, Phys. Rev. Lett. 107, 068701 (2011).

[32] J. P. Gleeson, Binary-State Dynamics on Complex Networks: Pair Approximation and Beyond, Phys. Rev. X 3, 021004 (2013).

[33] E. Cator and P. Van Mieghem, Second-order mean-field susceptible-infected-susceptible epidemic threshold, Phys. Rev. E 85, 056111 (2012).

[34] J. T. Matamalas, A. Arenas, and S. Gómez, Effective approach to epidemic containment using link equations in complex networks, Sci. Adv. 4, eaau4212 (2018).

[35] S. Weber and M. Porto, Generation of arbitrarily twopoint-correlated random networks, Phys. Rev. E 76, 046111 (2007).

[36] P. van Mieghem, Epidemic phase transition of the SIS type in networks, Europhys. Lett. 97, 48004 (2012).

[37] M. Boguñá, R. Pastor-Satorras, and A. Vespignani, Cut-offs and finite size effects in scale-free networks, Eur. Phys. J. B 38, 205 (2004).

[38] S. N. Dorogovtsev and J. F. F. Mendes, Evolution of networks, Adv. Phys. 51, 1079 (2002).

[39] W. Cota and S. C. Ferreira, Optimized Gillespie algorithms for the simulation of Markovian epidemic processes on large and heterogeneous networks, Comput. Phys. Commun. 219, 303 (2017).

[40] M. M. de Oliveira and R. Dickman, How to simulate the quasistationary state, Phys. Rev. E 71, 016129 (2005).

[41] R. S. Sander, G. S. Costa, and S. C. Ferreira, Sampling methods for the quasistationary regime of epidemic processes on regular and complex networks, Phys. Rev. E 94, 042308 (2016).

[42] M. Boguñá and R. Pastor-Satorras, Epidemic spreading in correlated complex networks, Phys. Rev. E 66, 047104 (2002).

[43] R. Pastor-Satorras and C. Castellano, Distinct types of eigenvector localization in networks, Sci. Rep. 6, 18847 (2016).

[44] S. B. Seidman, Network structure and minimum degree, Soc. Netw. 5, 269 (1983).

[45] R. Pastor-Satorras and C. Castellano, Eigenvector localization in real networks and its implications for epidemic spreading, J. Stat. Phys. 173, 1110 (2018).

[46] C. Castellano and R. Pastor-Satorras, Cumulative merging percolation and the epidemic transition of the susceptible-infectedsusceptible model in networks, arXiv:1906.06300v1. 
[47] C. Castellano and R. Pastor-Satorras, Competing activation mechanisms in epidemics on networks, Sci. Rep. 2, 371 (2012).

[48] S. C. Ferreira, R. S. Sander, and R. Pastor-Satorras, Collective versus hub activation of epidemic phases on networks, Phys. Rev. E 93, 032314 (2016).

[49] X. Huang and R. Durrett, The contact process on random graphs and Galton-Watson trees, arXiv:1810.06040v2.

[50] F. Chung, L. Lu, and V. Vu, Spectra of random graphs with given expected degrees, Proc. Natl. Acad. Sci. USA 100, 6313 (2003).
[51] T. Mountford, D. Valesin, and Q. Yao, Metastable densities for the contact process on power law random graphs, Electron. J. Probab. 18, 103 (2013).

[52] R. Pastor-Satorras and A. Vespignani, Epidemic dynamics in finite size scale-free networks, Phys. Rev. E 65, 035108(R) (2002).

[53] F. Radicchi, Predicting percolation thresholds in networks, Phys. Rev. E 91, 010801(R) (2015).

[54] F. Radicchi and C. Castellano, Breaking of the site-bond percolation universality in networks, Nat. Commun. 6, 10196 (2015). 